\title{
Intelligent Monitoring System for Home Based on FRBF Neural Network
}

\author{
Dezhong Zheng, Yang Wang and Yan Wang \\ Measurement Technology and Instrumentation Key Lab of Hebei Province, \\ Yanshan University, Qinhuangdao 066004, China \\ qhdzdz@sina.com,wy_ysu@hotmail.com
}

\begin{abstract}
In accordance with the requirements for home security and safe guard, a new type of intelligent monitoring system is researched and developed. The system is established with CAN bus and wireless as the foundation. Multi-sensor technology is used to improve the alarm algorithm of the system, a new fuzzy neural network is put forward as the classifier. There are four layers in the network, and the input signals are temperature and the concentration of $\mathrm{CO}$, smoke and $\mathrm{CO}_{2}$, the output signal is the fire probability. Radial basis function $(R B F)$ is used as the fuzzy membership function. The principal component analysis (PCA) is used to extract the information of sensors and an observation window is used to extract the necessary information for neural network. Experiments show that the system can provide accurate detection results in a short time.
\end{abstract}

Keywords: intelligent monitoring system; information fusion; neural network; the duration of time window

\section{Introduction}

In literature [1], with the rapid development of electronic engineering, communication technology and computer science, powerful tools has been given to protect human life and property. To develop a practical, reliable, and low-cost monitoring system to realize the real-time monitor of illegal intrusion, fire, and gas leak has important social significance.

In literature [2], the traditional home monitor system is usually unit with single-family. When an emergency occurs, the system would send massage to the home owner or dial the phone that was set by the owner. But because of the uncertainty of distance, measures may not be taken in time. At the same time, most systems are leaving idle due to the high false alarm and failed alarm rates. In literature [3] and [4], with the development of city residential, the newly built villages have begun to adopt the unified property management. The new monitoring system is based on residential units for community construction and management, but because of factors such as price, only a few trigger sensors are set in the village. And there is no unified standard to combine the two kinds of monitoring system, resulting in repeated investment and cannot achieve effective protection.

When intrusion, fire or other emergency occurs in the room, the alarm signal should be transmitted by the communication network. One of the key problems is that the system must have high sensitivity and reliability. In order to improve the reliability of the system, the data fusion technology is used to the detection algorithm. Fire detection is studied as an example in this paper.

Data fusion technology is also called multi-sensor information fusion in literature [5]. By using the redundancy and complementarity of a plurality of sensors, to obtain complete description and make the decision according to the comprehensive treatment of certain rules of multidimensional information. The widely used data fusion algorithm is D-S evidence theory, Bayesian methods, neural networks, etc. Neural network is 
considered to be an accurate and robust data fusion algorithm that has been widely used in fire detection system.

The BP neural network and D-S evidence theory are connected according to the sequence in literature [6]. Firstly, the parameters of sensor are input into neural network for the preliminary judgment. Then the D-S evidence theory is used to get the final result. In literature [7], the rate of change is detected in the information layer, and RBF neural network and fuzzy system are connected in the second layer. The output of the system is mainly decided by neural network and the fuzzy system is used to avoid output deviation. Although the neural network is used in the two kinds of fire detection systems, the performance of different neural network model is not compared. In addition, to detect the rate of change can only know the trend of signal, but cannot determine whether the signal is larger than the steady-state value. If the change trend of the signal and the relationship between the steady-state values cannot be divided, the system would provide false alarms. In most detection systems, the original parameters of sensors are input to neural network directly. The generalization ability and the fault-tolerant ability of neural networks have not been fully applied.

Fire detection system based on data fusion mainly includes four processes. First, collect the data of the field. Second, preproceing data and wavelet transform is used to denoise the data. Time window is used to extract the necessary information. Third, Principal component analysis (PCA) is used to analyze the data and extract the feature as the input of neural network. In this paper, a fuzzy neural network is put forward as classifier. Last, performances of system on error rate, alarm time and stability, including BPNN, RBFNN and FRBFNN, are investigated.

\section{The Overall Hardware Structure of Monitoring System}

Monitoring and control system hardware structure is shown in figure 1 . When intrusion,

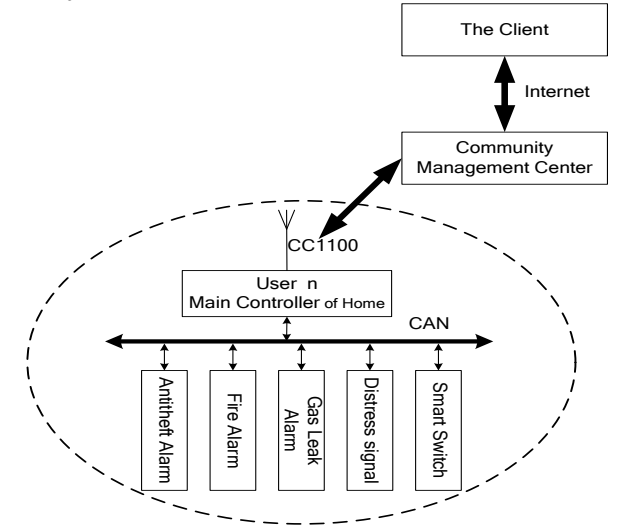

Figure 1. The Overall Hardware Structure of System

fire or other emergency occurs in home, the alarm signal is transmitted to the main controller through CAN bus, then to the management center by CC1100 wireless module (single hop or multi hop). The property management office takes measures in time, and passes alarm signal to users in the distance by the client software. In addition, the user can also switch the equipment in home through the client software.

Communication mode of the monitoring system can be divided into two major categories of wired and wireless. There are many sensor modules in home, so the cost would be high if wireless communication is taken. And now people pay more attention to electromagnetic radiation, wireless communication within the house may limit to be applied. So the communication network in the house should be embedded within the pipeline way. If the communication network between main controller and the community management center is adopted by wired, then cable furrow should be dug or open wire- 
line should be set up, which cost high and the communication quality decreased rapidly with the increase of distance. When the cable is laid, the size of communication capacity is hard to expand. The wireless communication costs less and can be expanded conveniently, so wireless is taken outside.

Within the house, the wired communication network consists of RS485, CAN, LonWorks protocol, etc. CAN bus is chosen as the through comparing the communication network in the house. Short distance wireless communication mode mainly include Bluetooth, WiFi, ZigBee, Wireless Radio Frequency (RF), etc. The communication distance of Bluetooth cannot meet the requirements. WiFi is suitable for higher bandwidth requirements of applications, and it has larger power consumption and higher cost. ZigBee has the advantages of low power consumption, but it's suitable for application when the battery as the power supply, its communication rate is only $250 \mathrm{kbps}$ and the distance of communication is short. The technology of RF is mature and the cost of RF is low. There are two kinds of schemes in the design of RF module: integrated RF module or adopt RF chip design. The first solution development cycle is short, but the price is higher. To design RF module, the development cycle is long, and the RF module involves the high frequency circuit design, if the design is unreasonable, the module performance will not meet the requirements.In addition, the RF module usually requires 4 or 6 layers PCB design, so the development cost of the first scheme may be higher. So the system uses the first scheme. The commonly used RF module is shown in table 1.

Table 1. The Comparison of Common RF Module

\begin{tabular}{ccccc}
\hline RF Chip & CC1100 & CC2500 & nRF903/905 & nRF24L01 \\
\hline Frequency & $433 \mathrm{MHz}$ & $2.4 \mathrm{GHz}$ & $433 \mathrm{MHz}$ & $2.4 \mathrm{GHz}$ \\
$\begin{array}{c}\text { Transmission } \\
\text { Rates }\end{array}$ & $500 \mathrm{kbps}$ & $500 \mathrm{kbps}$ & $50 \mathrm{kbps}$ & $2 \mathrm{Mbps}$ \\
$\begin{array}{c}\text { Transmission } \\
\text { Distance }\end{array}$ & $500 \mathrm{~m}$ & $200 \mathrm{~m}$ & $100 \mathrm{~m}$ & $100 \mathrm{~m}$ \\
\hline
\end{tabular}

Considering there are many obstacles in the community, $433 \mathrm{MHZ}$ which has strong ability of diffraction is chosen as the working band. CC1100 is better than nRF903/905 in the data transmission rate and communication distance, so CC1100 is chosen as the communication module of the system.

When an emergency occurs in the house, the system sends alarm to management center, and management center sends information to user. Remote communication scheme commonly used mainly include GSM, GPRS, TCP/IP, etc. TCP/IP protocol is the most stable way to transmit remote communication. The network architecture has two types: $\mathrm{C} / \mathrm{S}$ and $\mathrm{B} / \mathrm{S}$. $\mathrm{C} / \mathrm{S}$ needs to design two terminal communication software: the server and the client. The process of $\mathrm{C} / \mathrm{S}$ will be submitted to the server after tasking, which can help to reduce server load operation and realize the resource optimization. Only users that install the client use the system ensures the security of the software, so C/S architecture is chosen as the computer system.

\section{Fire Alarm System Based on Data Fusion}

This paper mainly study the sensitivity and reliability of the fire alarm system. Multisensor information fusion technique is used in fire alarm system, which can realize the fire alarming earlier, to reduce the error rate and improve the reliability of the system. The overall process is shown in Figure 2. 


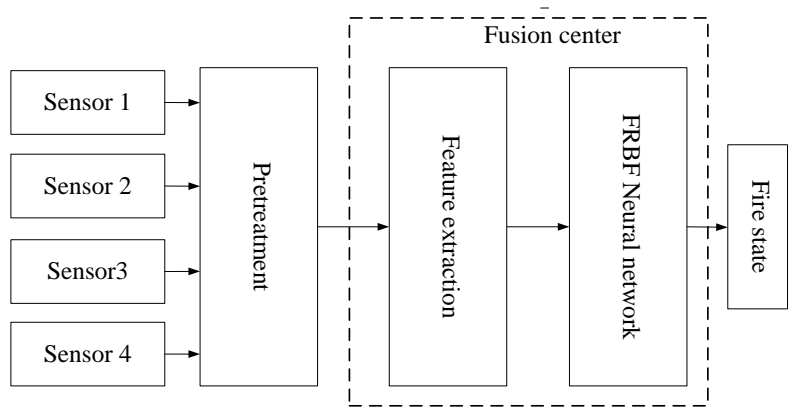

Figure 2. The Process of Fire Detection System

\subsection{Sensor Selection of Fire alarm System}

In order to achieve a comprehensive, effective monitoring of fire condition of the environment, a variety of sensors need to be set up. The selection of number and type of sensor decides weather the system can accurately identify the primary factor of fire. In literature [8] it is proved that the temperature, smoke, $\mathrm{CO}$ and $\mathrm{CO}_{2}$ sensors can show fire characteristics fully and accurately, and has good economic value. Therefore, the system chose sensors of temperature, smoke, $\mathrm{CO}$ and $\mathrm{CO}_{2}$ as fire detection module.

\subsection{PCA algorithm used for Feature Extraction}

The information collected by sensors usually contains a lot of noise. Therefore, the wavelet threshold method is used to deal with the noise. In fire detection, sensor sampling frequency is quite high. But the fire occurres only by chance. Therefore, the characteristic signal must be processed before send into neural network. In order to study the change trend of characteristic parameters in a certain period of time. A time window is used to extract the characteristic information of a period of time. The duration and step size of time window directly affects the accuracy of system in literature [8]. The length of time window is more important than step size. The duration of the time window is $60 \mathrm{~s}$. When the neural network is trained, the window is moved in steps of 250s and when the neural network is detected the window should be moved in steps of 50s. While the time window is easy to cause the high dimension of data fusion, PCA is used to extract the feature of data.

Feature extraction of the fire characteristic parameters by PCA, and the training sample set $F$ to calculate the mean vector of training samples $\bar{x}$. Construct the overall walk matrix $\sum_{r}(N \times N$ matrix $)$, that is

$$
\sum_{r}=\frac{1}{M} \sum_{k=1}^{M}\left(x_{k}-\bar{x}\right)\left(x_{k}-\bar{x}\right)^{T}
$$

Where $\bar{x}=\frac{1}{M} \sum_{k=1}^{M} x_{k}$, according to this, the projection function is defined as:

$$
J(U)=\left|U^{T} \sum_{r} U\right|
$$

Operator $|\cdot|$ strives for the determinant of a matrix. Accordingly, projection criterion is defined as:

$$
\begin{aligned}
& J\left(U_{\text {opt }}\right)=\max _{U}(J(U))=\max _{U}\left|U^{T} \sum_{r} U\right| \\
& U_{\text {opt }}=\arg \max _{U}\left|U^{T} \sum_{r} U\right|
\end{aligned}
$$

Through this the optimal projection matrix $U_{\text {opt }}$ is determined. This process can be transformed into solving the characteristic equation

$$
\lambda(u)=\sum_{r} u
$$


The eigenvalue $\lambda$ and the eigenvectors $u$ corresponding to it. $U_{o p t}$ is made up of the first $r$ maximum value that the eigenvectors $U_{\text {opt }}=\left[u_{1}, u_{2}, \cdots, u_{r}\right]$ corresponding to the eigenvalue of $\sum_{r}$.

Assume that the input matrix $x \in R^{N}$, its main components

$$
y=U_{\text {opt }}^{T} x=\left[y_{1}, y_{2}, \cdots, y_{r}\right]^{T}
$$

Where $y \in R^{r \times 1}$, because $N$ will increase with $\Sigma_{r}$, the high computational complexity of formula (10). As a result, singular value decomposition SVD is usually used to simplify the solving process. If $\delta_{k}=\left(x_{k}-\bar{x}\right), \quad A=\frac{1}{\sqrt{M}}\left[\delta_{1}, \delta_{2}, \cdots, \delta_{M}\right]$ , $A=\in R^{N \times M}$, formula (6) can be change to

$$
\sum_{r}=\frac{1}{M} \sum_{k=1}^{M} \delta_{k} \delta_{k}^{T}=A A^{T}
$$

According to SVD theorem, $A A^{T}$ and $A^{T} A$ has the same eigenvalue $\lambda$. And if $\Sigma^{\prime}=A^{T} A$, we can know that $\Sigma_{r}$ and $\Sigma^{\prime}$ has the same eigenvalue $\lambda$.

The eigenvalue and the eigenvectors corresponding to it of matrix $\sum_{r}$ order $N$ can get by evaluat the eigenvalue $\lambda$ and the eigenvectors $u$ of matrix $\Sigma^{\prime}$ order $M$. That is:

$$
U=A V D^{-1 / 2}=\left[u_{1}, u_{2}, \cdots, u_{r}\right]
$$

Where $u_{i}=\frac{1}{\sqrt{\lambda_{i}}} A v_{i}, \quad i=1,2, \cdots, r$.

Then the cumulative contribution rate characteristic value of $B_{1}, B_{2}, \cdots, B_{n}$ needs to be calculated. When the cumulative contribution rate $B_{k} \geq 85 \%$, the principal components $\alpha_{1}, \alpha_{2}, \cdots, \alpha_{k}$ of the first $K$ is extracted as the main element. And in order to ensure the accuracy of the neural network, $K$ should be not less than four.

\subsection{Extract the Characteristics by Fuzzy Neural Network}

Fire classification is completed by fuzzy neural network, the network module is composed of input fuzzy, fuzzy rules and output defuzzification. Fuzzy neural network proposed in this paper has two hidden layer and it is feed forward neural networks composed by 4 layers. The first layer is input layer, by four neurons respectively represent temperature, smoke, $\mathrm{CO}$ and $\mathrm{CO}_{2}$ concentration of the input, the second is blurred layer, the third layer is fuzzy rules calculations, the fourth layer to output layer. The structure of the fuzzy neural network is shown in figure 3.

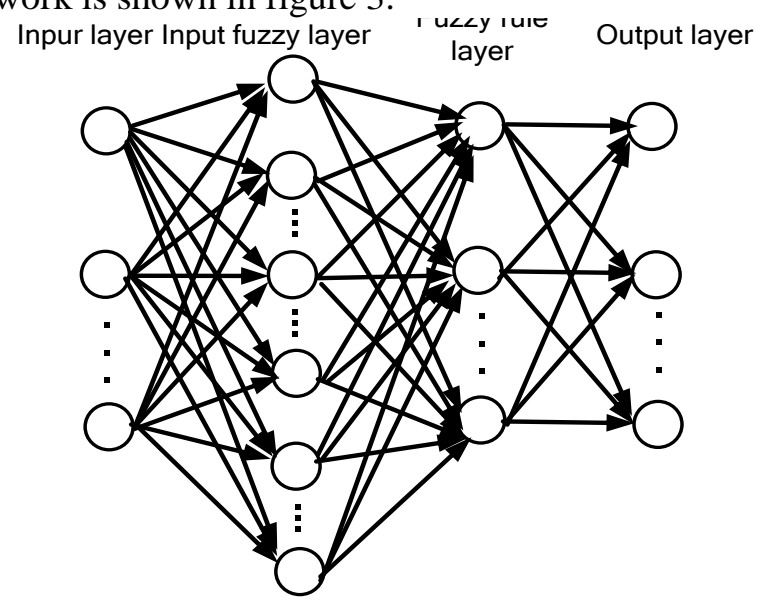

Figure 3. The Structure Diagram of FRBFNN 
The first layer is the input layer, each input neuron is connected directly, and node number and dimension of input vector is same. If the input neurons of $S$ are $X_{1}, X_{2}, \cdots, X_{s}$, the nodes of this layer can be expressed as:

$$
f_{1}(i)=X_{i}=\left[x_{1}^{i}, x_{2}^{i}, \cdots, x_{n}^{i}\right]^{T}
$$

Where $n$ stand for the sample size and $i=1,2,3,4$.

The second layer is the input fuzzy layer, the gauss radial basis function (RBF) is used for fuzzy membership function membership $f_{2}(i, j)$ of each input variable.

$$
f_{2}(i, j)=\exp \left\{-\frac{\left(f_{1}(i)-c_{i j}\right)^{2}}{\left(\sigma_{i j}\right)^{2}}\right\}
$$

Where $c_{i j}$ and $\sigma_{i j}$ represent the mean value and standard of the input variables of $i$ and the fuzzy set membership function $j$. If the layer has eight nodes, which means the input signals are quantified by two nodes, the corresponding fuzzy sets of $\{1,0\}$. Elements in the collection are: Fire (L) and Not Fire (O). The third layer is the fuzzy rule layer, to carry out comprehensive treatment of characteristic para meters, the fuzzy rules are mapped to the output space, fuzzy reasoning and control rules storage. The total number of nodes is fuzzy rule number, by that:

$$
c=a^{b}
$$

Where $a$ is the number of fire characteristic parameter, $b$ is the number of input variables that divided into state. $a$ is 4 and $b$ is 2 . Therefore, this layer should have 16 fuzzy rules. Each node represents a fuzzy rule, calculated as the product of all the nodes of the input signal of the fuzzy rules, that is:

$$
f_{3}(j)=\prod_{j=1}^{S} f_{2}(i, j), \quad S=\prod_{i=1}^{S} S_{i}
$$

Where $j=1,2, \cdots, t$.

The fourth layer is the blurred output layer, the center average defuzzification (the center average defuzzifier), as shown below:

$$
f_{4}(i)=y_{i}=\frac{\sum_{i=1}^{n_{i}} w_{i}^{\prime} \cdot \mu_{i}}{\sum_{i=1}^{n_{i}} \mu_{i}}, \quad i=1,2, \cdots, l
$$

$w_{i}^{\prime}$ is the weight function type of output layer, the $\mu_{i}$ membership functions for the input vector. When the fuzzy neural network is used, one of the main problems to be solved is the design of input and output. The input of the fuzzy neural network with 4 neurons, respectively by each sensor signal pre-processing. Output has 2 neurons: fire and not fire, respectively by two-dimensional orthogonal vector, as shown in table 2 . In the fire detection environment was tested by using fuzzy neural network, the values of output should be near zero and one. Zero point five is used as the fire and not fire condition critical value.

Table 2. The Output Design of Neural Network

\begin{tabular}{ccc}
\hline Output & Neuron One & Neuron Two \\
\hline Fire & 1 & 0 \\
Not Fire & 0 & 1 \\
\hline
\end{tabular}

\section{Data Selection and Experiment Analysis}

In order to study the performance of different sensors in the fire detection system, USA National Institute of standards and Technology (NIST) carried out a series of 
experiments in specifically built residential buildings in literature [10]. Where a construction area of eighty-five square meters, there are three bedrooms, a bathroom, a kitchen and a living room. The internal structure of the building fire is shown in figure 4 .

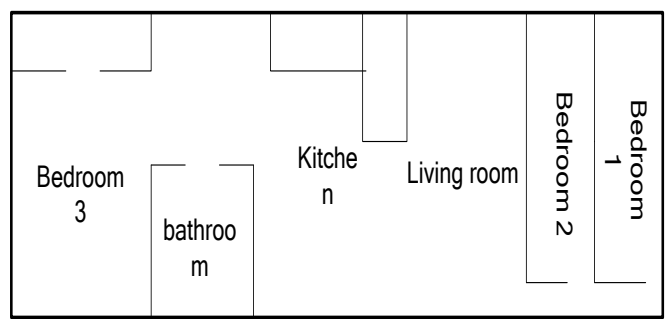

Figure 4. The Internal Structure of Fire Research Building

Experiment SDC31 is selected randomly for follow-up study, the sofa in the living room is the burning material, details of burning material can be found in literature [10], and ignited by smoldering fire, data of bedroom 2 is selected to study. According to needs, the data of temperature, smoke, $\mathrm{CO}$ and $\mathrm{CO}_{2}$ sensors are taken. The temperature sensor is thermocouple sensor, smoke concentration by laser equipment based on the measured extinction principle, concentration of $\mathrm{CO}$ and $\mathrm{CO}_{2}$ by infrared gas analyzer (NDIR) measurement. The equipment are subjected to rigorous correction before the experiment, the installation position are $20 \mathrm{~mm}$ under the ceiling.

The original parameter of each sensor is shown in Figure 5.

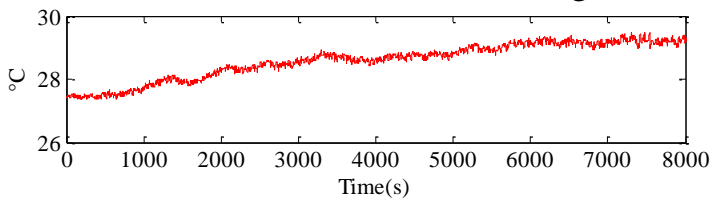

(a) The curves of temperature changing with time

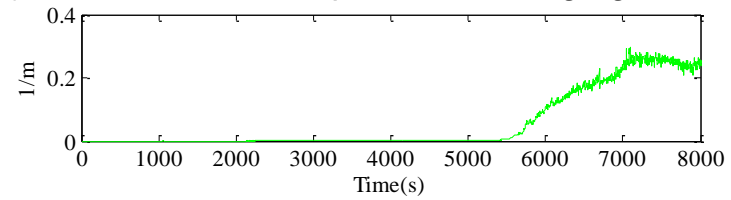

(b) The curves of smoke concentration changing with time

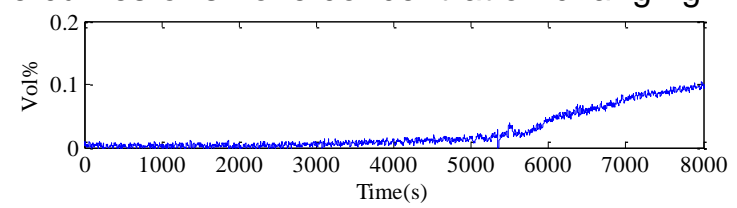

(c) The curves of $\mathrm{CO}$ concentration changing with time

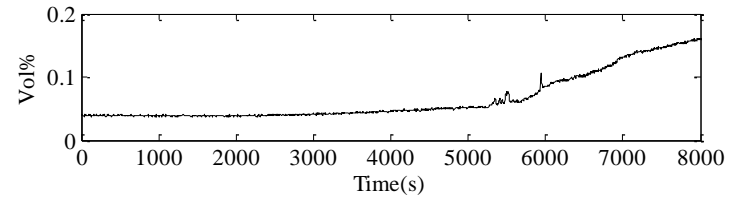

(d) The curves of $\mathrm{CO} 2$ concentration changing with time

Figure 5. The Raw Data of SDC31 Experiment

\section{Experimental Results and Analysis}

The experiment is implemented in MATLAB. Performances of system on error rate, alarm time and stability, including BPNN, RBFNN and FRBFNN, are compared. For the stability of the system, three kinds of neural network were repeated ten times. Fire alarming time should be 1900 s by observing the original data in fire state point, and 
the neural network should have the ability to distinguish the state near the fire. Therefore, the fire alarming time allowed having small differences. But the minimum value shall not be less than 1700 s, otherwise the error rate of the system is too high.

The data after wavelet denoising is shown in figure 6 .

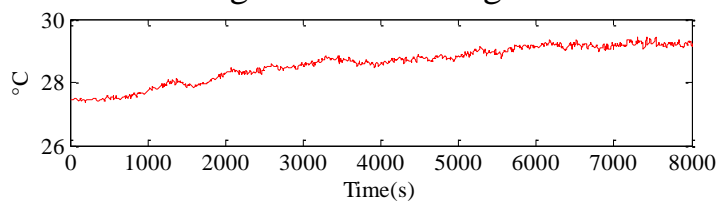

(a) The curves of temperature changing with time

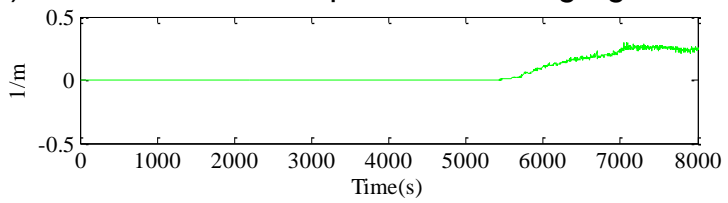

(b) The curves of smoke concentration changing with time

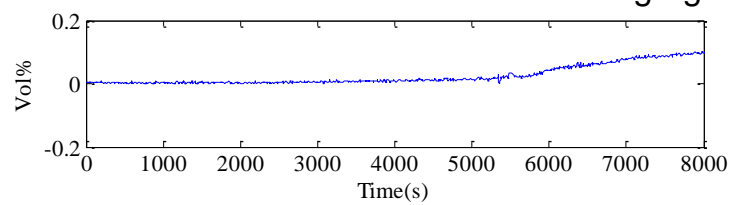

(c) The curves of $\mathrm{CO}$ concentration changing with time

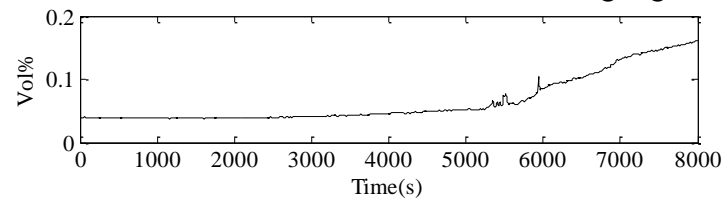

(d) The curves of $\mathrm{CO} 2$ concentration changing with time

Figure 6. The Data After Wavelet Denoised

Then data needs to be normalized before feature extraction by the formula:

$$
\bar{x}_{i}=\frac{x_{i}-x_{\min }}{x_{\max }-x_{\min }}
$$

And Extract data by time window for next research, as shown in figure 7 .

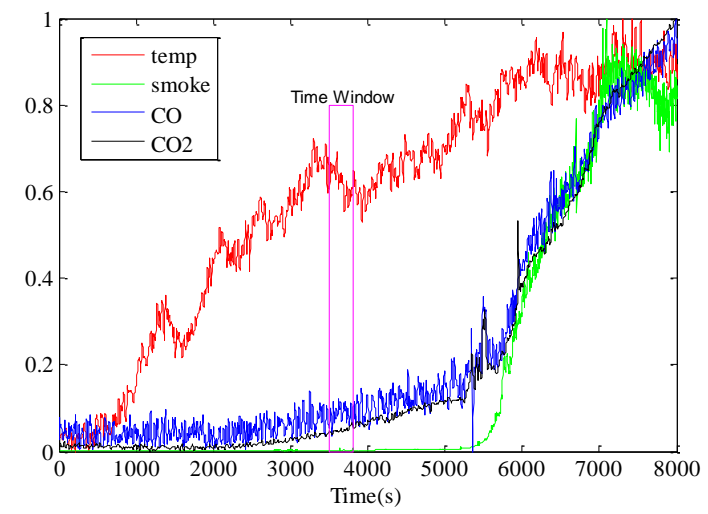

Figure 7. The Data After Normalization

Then principal component of data is extracted by PCA and input respectively to three different network models for learning and training. Test data are input to the network, the results are shown in figure 8 , figure 9 and figure 10 . The results of three repeated experiments of BP and FRBF neural network respectively are given. 
The results of three repeated experiments of RBF neural network are exactly same, so only one result is listed.

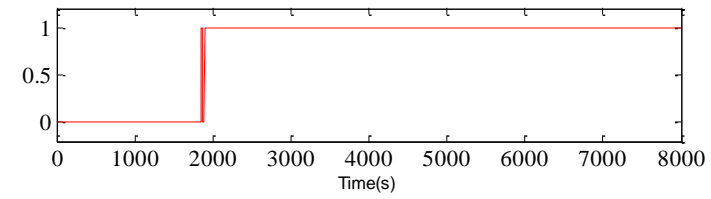

(a) The result of the first experiment of BP neural network

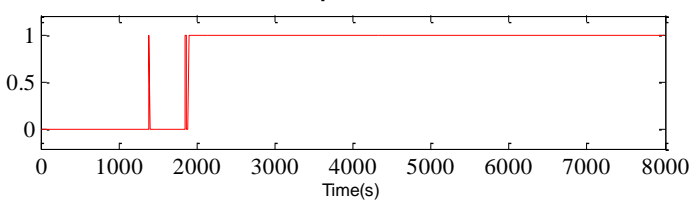

(b) The result of the second experiment of BP neural network

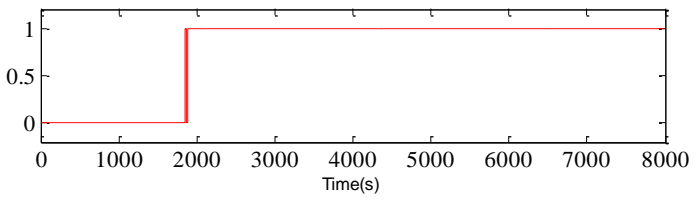

(c) The result of the third experiment of BP neural network

Figure 8. The Prediction Result of BP Neural Network

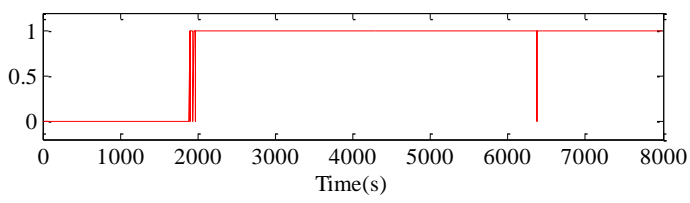

Figure 9. The Prediction Result of RBF Neural Network

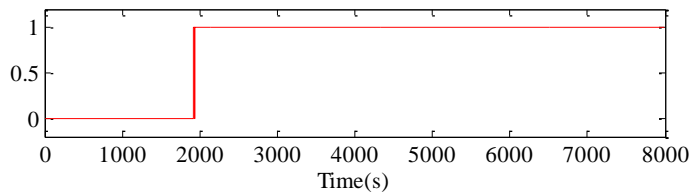

(a) The result of the first experiment of FRBF neural network

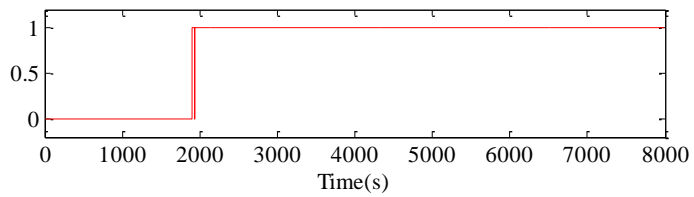

(b) The result of the second experiment of FRBF neural network

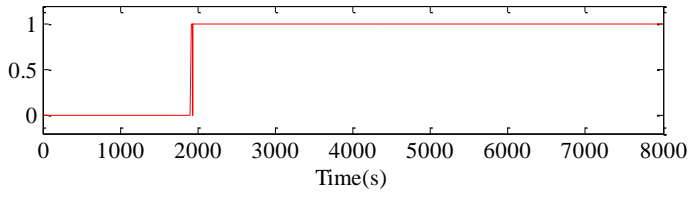

(c) The result of the third experiment of FRBF neural network

\section{Figure 10. The Prediction Result of RBF Neural Network}

\subsection{Sensitivity Analysis}

Fire alarming time of three different network models in ten repeated experiments are shown in figure 11. Fire alarming times of RBF and FRBF neural network in ten repeated experiments are almost remain the same. And their sensitivity is close to each other. Fire alarming time of BP network fluctuates a lot. Therefore, when the 
system adopts RBF or FRBF neural network as the classifiers, it could have better sensitivity for fire alarming time.

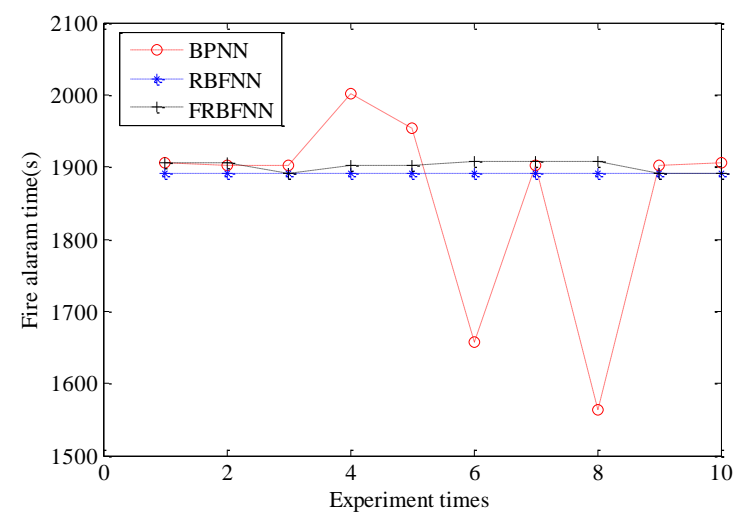

Figure 11. Fire Alarming Time of Ten Repeated Experiments

\subsection{Reliability Analysis}

The reliability of the system is determined by false alarm and alarm failure. Error rate is the result of false alarm and alarm failure together, Figure 12 shows error rate of three kinds of neural networks in ten repeated experiments. The error rate of BP network fluctuates larger than the other neural networks, and there are two high error rates that beyond the acceptable range. Error rate of RBF network is stable at very low levels. Error rate of FRBF neural network fluctuates between BP and RBF neural networks, and they are smaller than error rate of RBF neural network. Therefore, FRBF neural network is better than BP and RBF network in reliability.

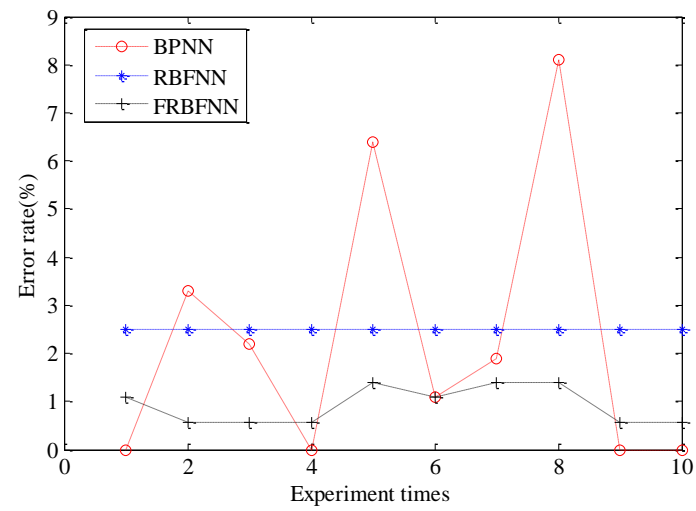

\section{Figure 12. Error Rate of Ten Repeated Experiments}

In addition, the stability of RBF neural network is the best through the analysis of sensitivity and reliability. The stability of FRBF neural network is after RBF neural network, the results of BP network are more random and its stability is the worst of three. In summary, the performance of FRBF neural network is the best. But the processor of FRBF may cost more money and the running time of the algorithm is longer than the other two. Therefore, the neural network should be chosen according to the requirement of application in practice.

\section{Conclusion}

A new type of intelligent monitoring system is researched in this paper. When an emergency occurs, the alarm signal would be sent to property management and family members through the network. In order to improve the sensitivity and reliability of 
system, data fusion technology is applied in the alarm system. A fuzzy neural network is put forward as the classifier and the performance are compared with BP and RBF neural networks. In order to guarantee the accuracy of the system, data of USA NIST was used to do the testing in fire alarm. As a result, the degree of prediction accuracy is increased and the degree of error rate is decreased. The system can be used not only in the intelligent monitoring system, but also in the field of intelligent building, etc. It has very good application prospects.

\section{References}

[1] S. H. Lee, J. G. Lee and M. Kyung, "Smart Home Security System Using Multiple ANFIS", International Journal of Smart Home, vol. 7, no. 3, (2013).

[2] H. Q. Wang and Y. Cheng, "Development of intelligent household alarm system based on LabVIEW", J. Wuhan Inst. Tech, vol. 33, no. 11, (2011).

[3] J. Peng and L. X. Lu, "Design of Village monitoring System Based on Lonworks Fieldbus", Journal of Nanchang University (Engineering \& Technology), vol. 33, no. 3, (2011).

[4] X. H. Liu and Z. Tian, "Design and implementation for intelligent security and defense system based on internet of things", Manufacturing Automatin, vol. 34, no. 17, (2012).

[5] H. Fan, L. Gao and L. J. Zhou, "AppIication of PFTTH technology in smart grid", Electric Power Automation Equipment, vol. 33, no. 7, (2013).

[6] X. J. Zhang, "Fire alarm system based on BP neural network and D-S Evidence Theory", Instrument Technique and Sensor, vol. 1, (2011).

[7] Y. X. Liu and X. X. Yuan, "Applications of fuzzy reasoning techniques and neural network in fire detection system", Computer Measurement \& Control, vol. 21, no. 12, (2013).

[8] X. G. Wang, S. M. Lo and H. P. Zhang, "Influence of Feature Extraction Duration and Step Size on ANN based Multisensor Fire Detection Performance", Procedia Engineering, vol. 52, (2013).

[9] L. A. Cestari, C. Worrell and J. A. Mike, "Advanced fire detection algorithms using data from the home smoke detector project”, Fire Safety Journal, vol. 40, no. 1, (2005).

[10] R. W. Bukowski, R. D. Peacock and J. D. Averill, "Performance of Home Smoke Alarms: Analysis of the Response of Several Available Technologies in Residential Fire Settings, National Institute of Standards and Technology”, Fire Readearch Division, Building and Fire Research Laboratory, (2008).

\section{Authors}

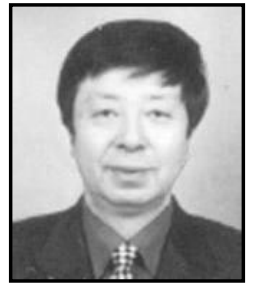

Dezhong Zheng, born 1949. Professor and tutor of ph.d. student, Yanshan University. His main research directions are smart home, multisensor information fusion, system simulation, signal processing.

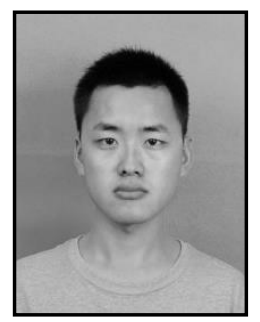

Yang Wang was born in July 1990. He holds a bachelor's degree and now is majoring in Yanshan University for his master degree. His main research directions are smart home and multisensor information fusion.

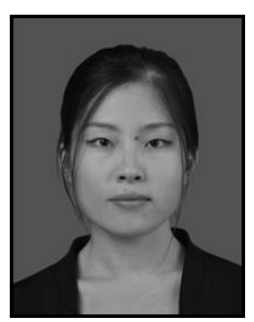

Yan Wang received her master's degree in Measuring and Testing Technologies and Instruments from Yanshan University, she is currently a university teacher in Baoding, hebei province , Baoding college. 
International Journal of Smart Home

Vol. 9, No. 2 (2015) 